Imperial/TP/95-96/26

hep-th/9602064

\title{
Self-duality of Born-Infeld action and Dirichlet 3-brane of type IIB superstring theory
}

\author{
A.A. Tseytlin ${ }^{\star \dagger}$ \\ Theoretical Physics Group, Blackett Laboratory, \\ Imperial College, London SWY 2BZ, U.K.
}

\begin{abstract}
$D$-brane actions depend on a world-volume abelian vector field and are described by Born-Infeld-type actions. We consider the vector field duality transformations of these actions. Like the usual $2 \mathrm{~d}$ scalar duality rotations of isometric string coordinates imply target space $T$-duality, this vector duality is intimately connected with $S L(2, Z)$-symmetry of type IIB superstring theory. We find that in parallel with generalised 4-dimensional Born-Infeld action, the action of 3-brane of type IIB theory is $S L(2, Z)$ self-dual. This indicates that 3-brane should play a special role in type IIB theory and also suggests a possibility of its 12-dimensional reformulation.
\end{abstract}

February 1996

\footnotetext{
* e-mail address: tseytlin@ic.ac.uk

$\dagger$ On leave from Lebedev Physics Institute, Moscow.
} 


\section{Introduction}

Type II superstring theories have supersymmetric $p$-brane solitonic solutions supported by Ramond-Ramond (R-R) sources [1,2,2,3]. These solitons have alternative description in terms of open strings with Dirichlet boundary conditions. $D$-branes [4,5,6] provide an important tool [7] to study non-perturbative properties [8,9] of superstring theories (see, e.g., [10,11,12, 13, 14, 15, 16, 17]). They are described by an effective Dirac-Born-Infeld (DBI) action [5] (supplemented with extra couplings to R-R fields [11, 15, 18, 19, 20]) which is closely connected to the Born-Infeld (BI) type effective action of open string theory [21,22, 23, 24, 25, 26].

The (bosonic part of) the DBI action of a $D$-p-brane is given by the $d=p+1$ dimensional world-volume integral and depends on the embedding coordinates $X^{\mu}$ and the world-volume vector vield $A_{m}$. Our aim below will be to consider in detail the case of the $D$-3-brane of type IIB theory which (like the 2-brane in type IIA case) should play a special role in this theory. The corresponding action can be interpreted as a generalisation of $d=4 \mathrm{BI}$ action coupled to a special background metric, dilaton, axion, etc. Using the remarkable fact that, like Maxwell action, the $d=4 \mathrm{BI}$ action is invariant [27,28,29] under the semiclassical vector duality transformation $\left(A_{m} \rightarrow \tilde{A}_{m}\right)$, we shall demonstrate that the $D$-3-brane action (combined with type IIB effective action) is invariant under the $S L(2, Z)$ symmetry of type IIB theory [30,8,96. 0 . This is consistent with expectations [33, 10] that while there are $S L(2, Z)$ multiplets of type IIB string solutions, the self-dual 3-brane solution [1.2] should be unique.

As in the case of the relation between the scalar-scalar world-sheet duality and $T$ duality of string theory, we suggest that the world-volume vector duality of $D$-brane actions is a key to understanding of the $S L(2, Z)$ symmetry of type IIB theory. The presence in the $D$-3-brane action of a propagating world-volume vector field with 2 physical degrees of freedom (and analogy with $D$-2-brane related [18,19] to the 11-dimensional supermembrane [34]) points towards possible existence of a (non-Lorentz-covariant) 12-dimensional $D$-3brane formulation of type IIB theory.

1 This confirms the conjecture made in 31. The $S L(2, R)$ duality invariance of equations of motion following from a similar BI-dilaton-axion action was first proved in [32] but a connection of their action to string theory was unclear. The $D$-3-brane action invariant under $S L(2, R)$ symmetry of type IIB theory provides the proper context for the application of the result of [32]. 
As a preparation for a discussion of $D$-brane world-volume vector field duality transformations we shall first study (in Section 2) the semiclassical duality transformations of Born-Infeld actions in different dimensions.

The $D$-brane actions in type II theories may be found by computing the superstring partition function in the presence of a $D$-brane hypersurface which can be probed by virtual closed strings only through virtual open strings with ends attached to the surface [4]. In Section 3 we shall present a direct path integral derivation of the DBI action [5] which explains its relation to $D=10 \mathrm{BI}$ action. We shall then include the couplings to the background fields of R-R sector [15,19] and obtain the explicit form (of leading terms in derivative expansions) of $D$-p-brane actions for $p=1,2,3$.

The duality transformations of these actions will be considered in Section 4 . The cases of $p=1,2$ were previously discussed in [19,20] but our approach and interpretation is somewhat different. We shall find that while the action of $D$-string transforms covariantly under the $S L(2, R)$ duality of type IIB theory, the $D$-3-brane action is 'self-dual', i.e. is mapped into itself provided one also duality-transforms the $d=4$ world-volume vector field.

Some conclusions which follow from the analysis of $D$-brane actions will be presented in Section 5. In particular, we shall suggest that like the $D$-2-brane in type IIA theory, the $D$-3-brane plays a special role in type IIB theory. We shall also argue that the analogy with 2-brane case indicates that the dimension 'associated' with $D$-3-branes is 12 (different remarks on possible 12-dimensional connection of type IIB theory appeared in [35, 36, 37]).

\section{Duality transformations of Born-Infeld actions}

Before turning to $D$-brane actions let us discuss semiclassical duals of similar BornInfeld actions in different dimensions.

Dualities between various gaussian fields (scalar-scalar in $d=2$, scalar-vector in $d=3$, vector-vector in $d=4$, etc.) are, in general, true at the quantum (path integral level. Restricting consideration to semiclassical level duality transformations can be applied to more general non-gaussian actions depending only on field strengths. Such 'semiclassical duality' is a relation between the two actions which lead to dual sets of classical equations and have the same 'on-shell' value. Suppose $L(F)$ is a Lagrangian that depends on a $n$ form field in $d$ dimensions only through its field strength. Then $L^{\prime}(F, \tilde{A})=L(F)+i F d \tilde{A}$ where $\tilde{A}$ is a $\tilde{n}$-form field $(\tilde{n}=d-n-2)$, is equivalent to $L(d A)$. If $L$ is an algebraic 
function of $F$ (i.e. it does not depend on derivatives of $F$ ) then solving $\partial L^{\prime} / \partial F=0$ for $F$ and substituting the solution into $L^{\prime}$ will give a local 'dual' Lagrangian $\tilde{L}(d \tilde{A})$. 2

In the special case of $n=\tilde{n}=\frac{1}{2}(d-2)(n=0$ in $d=2, n=1$ in $d=4, n=2$ in $d=6$, etc.) it may happen that for some $L$ its 'semiclassical dual' $\tilde{L}$ is the same function of $d \tilde{A}$ as $L$ is of $d A$. Such $L$ can be called 'self-dual' in the sense that that duality maps $L(d A)$ into $L(d \tilde{A})$ (in general, up to an additional transformation of 'spectator' background fields). The usual gaussian choice $L=F^{2}$ is of course of that type. A remarkable non-trivial example of a semiclassically self-dual Lagrangian is the Born-Infeld Lagrangian in four dimensions [27,28,29].3

Let us start with the Born-Infeld action [40] in its simplest flat-space form (generalisations to the presence of other fields and couplings are straightforward)

$$
S_{d}=\int d^{d} x \sqrt{\operatorname{det}\left(\delta_{m n}+F_{m n}\right)}, \quad F_{m n}=\partial_{m} A_{n}-\partial_{n} A_{m}
$$

where we assume the $d$-dimensional space to have euclidean signature. To perform the semiclassical duality transformation we add the Lagrange multiplier term $\left(\Lambda^{m n}=-\Lambda^{n m}\right)$

$$
S_{d}=\int d^{d} x\left[\sqrt{\operatorname{det}\left(\delta_{m n}+F_{m n}\right)}+\frac{1}{2} i \Lambda^{m n}\left(F_{m n}-2 \partial_{m} A_{n}\right)\right],
$$

and first solve for $A_{m}$, finding that

$$
\begin{gathered}
d=2: \Lambda^{m n}=\epsilon^{m n} \Lambda_{0}, \quad \Lambda_{0}=\mathrm{const} ; \quad d=3: \quad \Lambda^{m n}=\epsilon^{m n k} \partial_{k} \tilde{A} ; \\
d=4: \Lambda^{m n}=\epsilon^{m n k l} \partial_{k} \tilde{A}_{l} ; \quad d \geq 5: \Lambda^{n k}=\frac{1}{(d-3) !} \epsilon^{n k m_{1} \ldots m_{d-2}} \partial_{m_{1}} \tilde{A}_{m_{2} \ldots m_{d-2}} .
\end{gathered}
$$

Then we solve for $F_{m n}$ and get the dual action $\tilde{S}_{d}(\tilde{F}), \tilde{F}=d \tilde{A}$.

The BI action is semiclassically equivalent to

$$
S_{d}(F, V)=\int d^{d} x\left[\frac{1}{2} V \operatorname{det}\left(\delta_{m n}+F_{m n}\right)+\frac{1}{2} V^{-1}\right]
$$

2 All such dualities are symmetries of equations of motion combined with Bianchi identities, or maps between one action and a dual one (with equivalent sets of equations of motion). To have duality as a manifest symmetry of a (non-Lorentz-invariant) action one is to double the number of field variables by introducing the 'dual fields' on the same footing as the original fields [6] [38, 39].

3 More general self-dual $d=4$ vector Lagrangians $L(F)$ should satisfy a functional constraint which is a 1-st order Hamilton-Jacobi-type partial differential equation. It has a family of solutions parametrised by one function of one variable [29]. The construction of non-gaussian self-dual actions for higher $n=\tilde{n}=\frac{1}{2}(d-2)$ forms (e.g. for $n=2$ in $d=6$ ) is, in principle, straightforward; in particular, there exists the direct generalisation of the BI action to $n>1, d=2 n+2$ [29]. 
where $V(x)$ is an auxiliary field. Using (2.4) leads to a slight simplification in two and three dimensions since for $d=2,3 \operatorname{det}\left(\delta_{m n}+F_{m n}\right)=1+\frac{1}{2} F_{m n} F^{m n}$ so that (2.4) is quadratic in $F$ and solving for $F$ in the analogue of (2.2) is straightforward. One ends up with

$$
\tilde{S}_{2,3}=\int d^{d} x\left[\frac{1}{2} V+\frac{1}{2} V^{-1}\left(1+\frac{1}{2} \Lambda^{m n} \Lambda_{m n}\right)\right],
$$

or, after elimination of $V$,

$$
\begin{gathered}
\tilde{S}_{2,3}=\int d^{d} x \sqrt{1+\frac{1}{2} \Lambda^{m n} \Lambda_{m n}}=\int d^{d} x \sqrt{\operatorname{det}\left(\delta^{m n}+\Lambda^{m n}\right)} \\
\tilde{S}_{2}=\int d^{2} x \sqrt{1+\Lambda_{0}^{2}} \\
\tilde{S}_{3}=\int d^{3} x \sqrt{1+\partial_{m} \tilde{A} \partial^{m} \tilde{A}}=\int d^{3} x \sqrt{\operatorname{det}\left(\delta_{m n}+\partial_{m} \tilde{A} \partial_{n} \tilde{A}\right)}
\end{gathered}
$$

Thus the dual to BI action in $d=2$ is a constant (or a 'cosmological term') [41], while the dual in $d=3$ is a membrane-type action for a scalar field.

Remarkably, the second form of the dual action in (2.6) is true also in $d=4,5$. A simple way to show this is to note that since the Lagrangian is Lorentz $(O(d))$ invariant algebraic function of $F_{m n}$ 'integrating out' $F_{m n}$ can be done (at each point $x$ ) in a special Lorentz frame where $F_{m n}$ is block-diagonal with eigen-values $f_{1}, \ldots, f_{[d / 2]}$. Then $\operatorname{det}\left(\delta_{m n}+F_{m n}\right)=\left(1+f_{1}^{2}\right) \ldots\left(1+f_{[d / 2]}^{2}\right)$ and we get from $(2.4)$

$$
L=\sqrt{\left(1+f_{1}^{2}\right) \ldots\left(1+f_{[d / 2]}^{2}\right)}+i \lambda_{1} f_{1}+\ldots+i \lambda_{[d / 2]} f_{[d / 2]}
$$

where $\lambda_{1}=\Lambda^{12}, \ldots$. In the case of $d=4,5 \operatorname{det}\left(\delta_{m n}+F_{m n}\right)=\left(1+f_{1}^{2}\right)\left(1+f_{2}^{2}\right)$ so that solving for $f_{1}, f_{2}$ and substituting back into the action gives

$$
\tilde{L}=\sqrt{\left(1+\lambda_{1}^{2}\right)\left(1+\lambda_{2}^{2}\right)}=\sqrt{\operatorname{det}\left(\delta^{m n}+\Lambda^{m n}\right)} .
$$

Thus the BI action in $d=4$ is 'self-dual', i.e.

$$
\tilde{S}_{4}=\int d^{4} x \sqrt{\operatorname{det}\left(\delta_{m n}+\tilde{F}_{m n}\right)}, \quad \tilde{F}_{m n}=\partial_{m} \tilde{A}_{n}-\partial_{n} \tilde{A}_{m}
$$

while in $d=5$ it is dual to the following antisymmetric tensor action

$$
\tilde{S}_{5}=\int d^{5} x \sqrt{\operatorname{det}\left(\delta^{m n}+H^{* m n}\right)}, \quad H^{* m n} \equiv \frac{1}{3 !} \epsilon^{m n k p q} H_{k p q}, \quad H_{k p q} \equiv 3 \partial_{[k} \tilde{A}_{p q]}
$$


The derivation of the action dual to $d=4 \mathrm{BI}$ action can be generalised to the case of the presence of an extra axion coupling term in (2.1),

$$
S_{4}=\int d^{4} x\left[\sqrt{\operatorname{det}\left(\delta_{m n}+e^{-\frac{1}{2} \phi} F_{m n}\right)}+\frac{1}{8} i \epsilon^{m n k l} C F_{m n} F_{k l}\right],
$$

where $C$ is a background axion field and we have also introduced a background dilaton field $\phi$ with $e^{\frac{1}{2} \phi}$ playing the role of an effective gauge coupling constant. The analogue of (2.7) then is

$$
L_{4}=\sqrt{\left(1+e^{-\phi} f_{1}^{2}\right)\left(1+e^{-\phi} f_{2}^{2}\right)}+i \lambda_{1} f_{1}+i \lambda_{2} f_{2}+i C f_{1} f_{2} .
$$

Solving for $f_{1}$ and $f_{2}$ one finds

$$
\begin{gathered}
f_{1}=-i \Delta^{-1}\left(\sqrt{\frac{\Delta+\lambda_{2}^{2}}{\Delta+\lambda_{1}^{2}}} \lambda_{1}-i e^{\phi} C \lambda_{2}\right), \\
\tilde{f}_{2}=-i \Delta^{-1}\left(\sqrt{\frac{\Delta+\lambda_{1}^{2}}{\Delta+\lambda_{2}^{2}}} \lambda_{2}-i e^{\phi} C \lambda_{1}\right), \quad \Delta \equiv e^{-\phi}+e^{\phi} C^{2} .
\end{gathered}
$$

The final expression for the dual action is then

$$
\begin{gathered}
\tilde{S}_{4}=\int d^{4} x\left[\sqrt{\left(1+e^{-\tilde{\phi}} \lambda_{1}^{2}\right)\left(1+e^{-\tilde{\phi}} \lambda_{2}^{2}\right)}+i \tilde{C} \lambda_{1} \lambda_{2}\right] \\
=\int d^{4} x\left[\sqrt{\operatorname{det}\left(\delta_{m n}+e^{-\frac{1}{2} \tilde{\phi}} \tilde{F}_{m n}\right)}+\frac{1}{8} i \epsilon^{m n k l} \tilde{C} \tilde{F}_{m n} \tilde{F}_{k l}\right],
\end{gathered}
$$

where

$$
e^{-\tilde{\phi}}=\frac{1}{e^{-\phi}+e^{\phi} C^{2}}, \quad \tilde{C}=-\frac{C e^{\phi}}{e^{-\phi}+e^{\phi} C^{2}},
$$

is the standard inversion $\tilde{\lambda}=-1 / \lambda, \lambda \equiv C+i e^{-\phi}$. Since the action (2.11) is also invariant under the shift $C \rightarrow C+q, q=$ const, the two transformations generate the full $S L(2, R)$ invariance group

$$
A_{m} \rightarrow \tilde{A}_{m}, \quad \lambda \rightarrow \tilde{\lambda}=\frac{p \lambda+q}{r \lambda+s}, \quad \lambda \equiv C+i e^{-\phi} .
$$

One can also replace $\delta_{m n}$ in (2.11) by a general metric $g_{m n}$ which should be invariant under $S L(2, R)$.

We conclude that the generalised BI action (2.11) is $S L(2, R)$-self-dual, i.e. its form is invariant under the vector duality accompanied by the $S L(2, R)$ transformation of the background fields $\phi, C$. Equivalent observation about the $S L(2, R)$ invariance of equations of motion that follow from this generalised axion-dilaton-BI action was made in [32]. As we shall see in Section 4.3, a similar action describes the dynamics of $D$-3-brane of type IIB superstring theory. 


\section{D-brane actions}

Below we shall first describe a direct path integral derivation of the D-brane actions explaining, in particular, the relation between the BI action of [21] and DBI action of [5]. While an understanding of a fundamental string action as a source added to the effective string field theory action needs a non-perturbative ('wormhole') resummation of string loop expansion [42], the open string description [4,7] of $D$-branes provides a simple perturbative recipe for deriving their actions.

Since $D$-branes are described by BI-type actions [5] we shall then (in Section 4) use the results of Section 2 to find how these actions change under vector duality transformations.

\subsection{Path integral derivation of D-brane action}

$D$-branes represent soliton configurations in superstring theory which have R-Rcharges [7]. Within string perturbation theory they are 'composite' objects which have effective 'thickness' of order $\sqrt{\alpha^{\prime}}$ 12, 13]. As the effective field theory action $S_{\text {eff }}$ for mass-

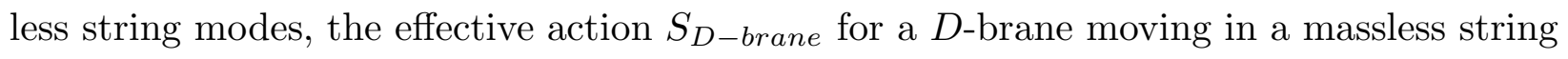
background is given by power series in $\alpha^{\prime}$. . The tree-level closed string effective action $S_{\text {eff }}$ can be represented in terms of ('renormalised') string partition on the sphere [43, 44]. In the case of the open string theory the effective action is given by the partition function on the disc [21,24,25 (loop corrections can be found by adding partition functions on higher genus surfaces [45]). In the superstring case the leading term in the disc partition function is finite (there is no quadratic $S L(2, R)$ Möbius volume infinity [25]) and is equal to the BI action.

Our aim is to find the $D$-brane action in a similar way by evaluating the string path integral in the presence of a $D$-brane. In view of the open string connection [4], the $D$-brane effective action (reconstructed in [5] from the equations of motion obtained from conformal invariance conditions [46,22]) can be derived directly by computing the corresponding string partition function on a disc. This is the partition function of virtual open strings with mixed Dirichlet-Neumann boundary conditions (i.e. with ends attached to a hyperplane) propagating in a condensate of massless string modes. The collective coordinates $X^{i}$ and internal vector $A_{m}$ degrees of freedom of the $D$-brane are represented

\footnotetext{
${ }^{4}$ In the case of a string ( $D=2$ effective theory) $\alpha^{\prime}$-corrections should be trivial up to a field
} redefinition, i.e. should affect only 'propagator' terms. At the same time, $D$-string is expected to become fundamental ('structureless') only after a resummation of string loops. 
by the boundary background couplings [4,5]. The combined action of the string massless modes and $D$-brane source can be represented as ( $t$ is a logarithm of $2 \mathrm{~d}$ cutoff)

$$
\begin{gathered}
S_{\text {eff }}(G, B, \phi ; C)+S_{D-\text { brane }}\left(X^{i}, A_{m} ; G, B, \phi ; C\right)=\left(\frac{\partial}{\partial t} Z_{\text {sphere }}\right)_{t=1}+Z_{\text {disc }}, \\
Z_{\text {disc }}=\int[d x d \psi] e^{-I}, \quad I=I_{M}+I_{\partial M}, \\
I_{M}=\frac{1}{4 \pi \alpha^{\prime}} \int d^{2} \xi\left[\left(\sqrt{g} g^{a b} G_{\mu \nu}+i \epsilon^{a b} B_{\mu \nu}\right)(x) \partial_{a} x^{\mu} \partial_{b} x^{\nu}+\alpha^{\prime} R^{(2)} \phi(x)\right]+\ldots, \\
I_{\partial M}=\frac{1}{2 \pi \alpha^{\prime}} \int d \tau\left[i A_{m}(x) \partial_{\tau} x^{m}+X_{i}(x) \partial_{\perp} x^{i}+\alpha^{\prime} e K \phi(x)\right]+\ldots .
\end{gathered}
$$

Here $C$ denotes the background $r$-form fields from the $\mathrm{R}$-R sector $\left(C_{1}, C_{3}\right.$ in type IIA and $C, C_{2}, C_{4}$ in type IIB theories) and dots stand for fermionic terms.5 The string coordinates $x^{\mu}(\mu=0,1, \ldots, 9)$ are split into $x^{m}(m=0, \ldots, p)$ and $x^{i}(i=p+1, \ldots, 9)$ with the boundary conditions $\left.\partial_{\perp} x^{m}\right|_{\partial M}=0,\left.x^{i}\right|_{\partial M}=0$ so that the boundary couplings depend only on $x^{m}$. An alternative possibility is to use the boundary condition $\left.x^{i}\right|_{\partial M}=\left.X^{i}\left(x^{m}\right)\right|_{\partial M}$ while setting $X_{i}$-coupling in (3.4) to zero [5]. This has an advantage of making obvious the interpretation of $X^{i}$ as the collective coordinates of $D$-brane but is less natural from the point of view of computing the vacuum partition function and also breaks the symmetry between $A_{m}$ and $X_{i}$ in $I_{\partial M}$. The two approaches are simply related to leading order of semiclassical expansion [5]: assuming $\left.x^{i}\right|_{\partial M}=\left.X^{i}\left(x^{m}\right)\right|_{\partial M}$ and making a shift $x^{i} \rightarrow x^{i}+$ $\left.X^{i}\right|_{\partial M}$ so that the new $x^{i}$ is subject to $\left.x^{i}\right|_{\partial M}=0$ one finds that $I_{\partial M}$ gets $X_{i}=G_{i j} X^{j}+\ldots$. In general the transformation between them is complicated and should involve redefinitions of background fields.

Choosing the flat $2 \mathrm{~d}$ metric (and ignoring the dilaton couplings) we can formally re-write the combined action $I$ as

$$
\begin{aligned}
I= & \frac{1}{4 \pi \alpha^{\prime}} \int d^{2} \xi\left(\left(\delta^{a b} G_{\mu \nu}+i \epsilon^{a b} B_{\mu \nu}\right)(x) \partial_{a} x^{\mu} \partial_{b} x^{\nu}\right. \\
& \left.+\partial_{a}\left[i \epsilon^{a b} A_{m}(x) \partial_{b} x^{m}+X_{i}(x) \partial^{a} x^{i}\right]+\ldots\right) .
\end{aligned}
$$

Let us first assume that the closed string couplings do not depend on $x^{i}$ (and are slowly varying in $x^{m}$ ). Then to compute the partition function (3.2) we may first do explicitly the

5 This description applies only to 'electric' $(p \leq 3) D$-branes which we shall consider in what follows. To generalise it to 'magnetic' ones one presumably is to double the number of the R-R fields in the action (cf. [38,39]). 
gaussian integration over $x^{i}$. Because of the absence of the zero mode of $x^{i}$ this will not produce the standard $\sqrt{\operatorname{det} G_{i j}}$ factor (usually present in the covariant $\sigma$-model measure). The only change will be in the couplings in the action (3.5) which will now depend on $d=p+1$ coordinates $x^{m}$ with standard Neumann boundary conditions. Expanding $x^{m}(\xi)=x_{0}^{m}+y^{m}(\xi)$ one can shift the $B\left(x_{0}\right) \partial y \partial y$ term to the boundary. Integrating first over the values of $y^{m}$ at the internal points of the disc and then over their boundary values one finds as in [21,45] that to the leading order in expansion in derivatives of fields $(d=p+1)$

$$
\begin{gathered}
Z_{d i s c}=c_{1} \int d^{d} x_{0} e-\phi \sqrt{\operatorname{det}\left(\hat{G}_{m n}+\hat{B}_{m n}+F_{m n}\right)}+\ldots \\
\hat{G}_{m n} \equiv G_{\mu \nu}\left(x_{0}\right) \partial_{m} X^{\mu} \partial_{n} X^{\nu}, \quad \hat{B}_{m n} \equiv B_{\mu \nu}\left(x_{0}\right) \partial_{m} X^{\mu} \partial_{n} X^{\nu} \\
X^{\mu} \equiv\left(x_{0}^{m}, G^{i j} X_{j}\left(x_{0}\right)\right), \quad \partial_{m}=\frac{\partial}{\partial x_{0}^{m}}, \quad F_{m n}=\partial_{m} A_{n}-\partial_{n} A_{m} .
\end{gathered}
$$

Equivalently, we may compute $Z_{\text {disc }}$ in a more '10d-symmetric' way by first performing the duality transformation 四 $x^{i} \rightarrow \tilde{x}_{i}$ in (3.5). Then the boundary term takes the standard open string form with $A_{\mu}=\left(A_{m}, X_{i}\right)$ so that the result is just the usual $D=10 \mathrm{BI}$ action for the $O(9-p, 9-p)$ dual background up to the factor (which as usual can be absorbed into the dilaton) accounting for the fact that $\tilde{x}_{i}$ does not have zero mode part

$$
\begin{gathered}
Z_{d i s c}=c_{1} \int d^{d} x_{0} e^{-\phi} \frac{1}{\sqrt{\operatorname{det}\left(\tilde{G}_{i j}+\tilde{B}_{i j}\right)}} \sqrt{\operatorname{det}\left(\tilde{G}_{\mu \nu}+\tilde{B}_{\mu \nu}+F_{\mu \nu}\right)}+\ldots, \\
\tilde{G}_{i j}+\tilde{B}_{i j}=\left(G_{i j}+B_{i j}\right)^{-1}, \quad \tilde{G}_{i m}+\tilde{B}_{i m}=\left(G_{i j}+B_{i j}\right)^{-1}\left(G_{j m}+B_{j m}\right), \ldots, \\
F_{m n}=\partial_{m} A_{n}-\partial_{n} A_{m}, \quad F_{m i}=\partial_{m} X_{i}, \quad F_{i j}=0 .
\end{gathered}
$$

The two expressions (3.7), (3.6) agree for constant $G, B$.

The background fields $G, B, \phi$ in (3.6) depend only on $x_{0}^{m}$. When their dependence on $x^{i}$ is taken into account in (3.5) one expects to find (3.6) with the fields depending on $X^{\mu}\left(x_{0}\right)=\left(x_{0}^{m}, X^{i}\left(x_{0}\right)\right)$. This indeed is what happens since one can re-introduce the zero mode for $x^{i}$ by the shift $x^{i} \rightarrow x^{i}+X^{i}\left(x_{0}\right)$ which eliminates the linear boundary coupling term $X_{i}\left(x_{0}\right) \partial_{\perp} x^{i}$.

The resulting $D$-p-brane action $S_{d}=Z_{d i s c}$ is the same as the DBI action of [5] (here $d=p+1$; in what follows we omit the index 0 on $x^{m}$ )

$$
S_{d}=\int d^{d} x e^{-\phi} \sqrt{\operatorname{det}\left(\hat{G}_{m n}+\mathcal{F}_{m n}\right)}+\ldots
$$




$$
\mathcal{F}_{m n}=F_{m n}+\hat{B}_{m n}, \quad \hat{G}_{m n}+\hat{B}_{m n}=\left(G_{\mu \nu}+B_{\mu \nu}\right)(X) \partial_{m} X^{\mu} \partial_{n} X^{n} .
$$

It depends only on transverse $D$-brane coordinates $X^{i}$ and on $A_{m}$, i.e. corresponds to the static gauge $X^{m}(x)=x^{m}$. The number of physical modes is the same for all values of $d=p+1: 10-d+d-2=8$, where $d-2$ corresponds to a vector in $d$ dimensions. In addition there are 8 fermionic modes as demanded by unbroken supersymmetry [7] (half of $N=2, D=10$ supersymmetry should be realised linearly and half - in a non-linear way). These on-shell degrees of freedom are the same as of $N=1, D=10$ Maxwell supermultiplet dimensionally reduced to $d$ dimensions [10].

It is natural to assume that the above action can be generalised to make it manifestly $D=10$ Lorentz and world-volume diffeomorphism invariant by relaxing the static gauge condition, i.e. treating all $X^{\mu}=\left(X^{m}, X^{i}\right)$ as 10 independent fields in $d$ dimensions. This will be assumed in what follows. 6

\subsection{Couplings to $R$ - $R$ background fields}

In addition to the above couplings (3.9) to NS-NS background fields $(G, B, \phi), D$-brane actions contain couplings to the background fields $C_{r}$ of R-R sector of type II theories. The leading-order couplings are effectively given by the fermionic zero mode $\left(\psi_{0}^{m}\right)$ factor and can be computed systematically [11, 15, 19, 20] using the techniques of [26]. Since the $\sigma$-model action contains the boundary term $\mathcal{F}_{m n} \psi^{m} \psi^{n}$ plus couplings to R-R fields $C_{r}$ the resulting leading-order term [15] has the following symbolic structure目 $\int d^{d} x_{0} d^{d} \psi_{0} \sum C_{r}\left(\psi_{0}\right)^{r} e^{\mathcal{F} \psi_{0} \psi_{0}}$. In addition, for 'magnetic' $D$-branes $(p \geq 3)$ the conformal invariance conditions imply that there should be a source term in the Bianchi identities for the field strengths $d C_{r}$ [0, [15, [19].

The explicit form of these coupling terms thus depends on particular $p$. For $p=$ $d-1=1,2,3$ one finds

$$
\begin{gathered}
S_{2}=\int d^{2} x\left[e^{-\phi} \sqrt{\operatorname{det}\left(\hat{G}_{m n}+\mathcal{F}_{m n}\right)}+\frac{1}{2} i \epsilon^{m n}\left(\hat{C}_{m n}+C \mathcal{F}_{m n}\right)+\ldots\right], \\
S_{3}=\int d^{3} x\left[e^{-\phi} \sqrt{\operatorname{det}\left(\hat{G}_{m n}+\mathcal{F}_{m n}\right)}+\frac{1}{2} i \epsilon^{k m n}\left(\frac{1}{3} \hat{C}_{k m n}+\hat{C}_{k} \mathcal{F}_{m n}\right)+\ldots\right],
\end{gathered}
$$

6 It is not clear, however, how this can be done in a manifestly supersymmetric way for $d \geq 4$ (see also Section 5).

7 One way to understand this expression is to note that the zero mode count on the disc implies that one of the R-R vertex operators should depend explicitly on the potential $C_{r}$ and not on its strength [16. 


$$
\begin{gathered}
S_{4}=\int d^{4} x\left[e^{-\phi} \sqrt{\operatorname{det}\left(\hat{G}_{m n}+\mathcal{F}_{m n}\right)}\right. \\
\left.+\frac{1}{8} i \epsilon^{m n k l}\left(\frac{1}{3} \hat{C}_{m n k l}+2 \hat{C}_{m n} \mathcal{F}_{k l}+C \mathcal{F}_{m n} \mathcal{F}_{k l}\right)+\ldots\right],
\end{gathered}
$$

where $\hat{C}_{m}, \hat{C}_{m n k}$ and $C, \hat{C}_{m n}, \hat{C}_{m n k l}$ are projections of the R-R fields of type IIA and type IIB theories $\left(\hat{C}_{m}=C_{\mu}(X) \partial_{m} X^{\mu}\right.$, etc. $)$ and dots stand for fermionic and higher-order terms. 8

These $D$-brane actions may be combined according to 3.1 with the effective action $S_{\text {eff }}$ for the massless string modes. For example, in the case of type IIB theory [30] $S_{\text {eff }}$ is given by 47,48

$$
\begin{gathered}
S_{\text {eff IIB }}=c_{0} \int d^{10} x \sqrt{G}\left(e^{-2 \phi}\left[R+4(\partial \phi)^{2}-\frac{3}{4}\left(\partial B_{2}\right)^{2}\right]\right. \\
\left.-\frac{1}{2}(\partial C)^{2}-\frac{3}{4}\left(\partial C_{2}-C \partial B_{2}\right)^{2}-\frac{5}{6} F^{2}\left(C_{4}\right)-\frac{1}{48} \frac{\epsilon_{10}}{\sqrt{G}} C_{4} \partial C_{2} \partial B_{2}+\ldots\right) .
\end{gathered}
$$

where $\partial B_{2}=\partial_{[\mu} B_{\nu \lambda]}$, etc., $F\left(C_{4}\right)=\partial C_{4}+\frac{3}{4}\left(B_{2} \partial C_{2}-C_{2} \partial B_{2}\right)$, and, following [48], it is assumed that the (conformally-invariant) self-duality constraint on $F\left(C_{4}\right)\left(F=F^{*}\right)$ is to be added at the level of equations of motion. This action is invariant under the $S L(2, R)$ symmetry [30,47]

$$
\begin{gathered}
g_{\mu \nu} \rightarrow g_{\mu \nu}, \quad \lambda \rightarrow \frac{p \lambda+q}{r \lambda+s}, \\
B_{\mu \nu} \rightarrow s B_{\mu \nu}-r C_{\mu \nu}, \quad C_{\mu \nu} \rightarrow p C_{\mu \nu}-q B_{\mu \nu}, \\
g_{\mu \nu} \equiv e^{-\frac{1}{2} \phi} G_{\mu \nu}, \quad \lambda \equiv C+i e^{-\phi},
\end{gathered}
$$

which is expected to be an exact duality symmetry of type IIB superstring theory [8,9, 33]. A natural question is whether this symmetry is present in the combined action of type IIB low-energy field theory and a $D$-brane source. As we shall see below, the combined equations of motion are indeed covariant under $S L(2, R)$ in the case of $D$-string and $D$ 3-brane. To demonstrate this it is necessary to perform the world-volume vector duality transformation similar to the one discussed above for BI action.

8 The constant $c_{1}$ in front of the actions (3.6),(3.9) (proportional to the dilaton tadpole on the disc) is assumed to be absorbed into $e^{\phi}$ and normalisations of the fields $C_{r}$. 


\section{Duality transformations of $D$-brane actions}

Since the $D$-brane actions (3.9)-(3.12) depend on $A_{m}$ only through $F_{m n}$ one can perform the semiclassical duality transformation as in the case of the BI actions in Section 2 , i.e. by adding the Lagrange multiplier term $\frac{1}{2} i \Lambda^{m n}\left(\mathcal{F}_{m n}-2 \partial_{m} A_{n}-\hat{B}_{m n}\right)$ and eliminating $\mathcal{F}_{m n}$ and $A_{m}$ from the action using their equations of motion. 9 The resulting dual action has equivalent set of equations of motion (with the roles of 'dynamical equations' and 'Bianchi identities' interchanged).

\subsection{D-string}

Starting with (3.10) we find that as in $(2.2),(2.3)$ the $A_{m}$-equation still implies $\Lambda=$ $\Lambda_{0}=$ const so that 19

$$
\tilde{S}_{2}=\int d^{2} x\left[\sqrt{e^{-2 \phi}+\left(\Lambda_{0}+C\right)^{2}} \sqrt{\operatorname{det} \hat{G}_{m n}}+\frac{1}{2} i \epsilon^{m n}\left(\hat{C}_{m n}-\Lambda_{0} \hat{B}_{m n}\right)\right] .
$$

The dual to $D$-string action can thus be interpreted as the standard fundamental string action in $S L(2, R)$ transformed metric and antisymmetric tensor background (cf.(3.14) with $\left.p=0, q=-1, r=1, s=\Lambda_{0}, \lambda \rightarrow-1 /\left(\lambda+\Lambda_{0}\right)\right)$

$$
\begin{gathered}
\tilde{S}_{2}=\int d^{2} x\left(\sqrt{\operatorname{det} \hat{G}_{m n}^{\prime}}+\frac{1}{2} i \epsilon^{m n} \hat{B}_{m n}^{\prime}\right), \\
G_{\mu \nu}^{\prime} \equiv \sqrt{e^{-2 \phi}+\left(\Lambda_{0}+C\right)^{2}} G_{\mu \nu}, \quad B_{\mu \nu}^{\prime} \equiv C_{\mu \nu}-\Lambda_{0} B_{\mu \nu},
\end{gathered}
$$

where $\hat{G}_{m n}=G_{\mu \nu} \partial_{m} X^{\mu} \partial_{n} X^{\nu}$, etc. This action (and its sum with (3.13)) is thus covariant under the type IIB $S L(2, R)$ transformations (3.15) of the background fields. Indeed, $G_{\mu \nu}^{\prime}$ can be written as $G_{\mu \nu}^{\prime}=\sqrt{\Delta\left(\Lambda_{0}\right)} g_{\mu \nu}, \quad \Delta\left(\Lambda_{0}\right)=e^{-\phi}+e^{\phi}\left(\Lambda_{0}+C\right)^{2}$, where $g_{\mu \nu}$ is the $S L(2, R)$-invariant Einstein-frame metric. Generic $S L(2, R)$ transformation shifts the parameter $\Lambda_{0}\left(\Lambda_{0}^{\prime}=s \Lambda_{0}+q\right)$ and also rescales the coefficient in front of the action (by factor $\left.p+r \Lambda_{0}\right) 10$ This duality covariance was not apparent before the duality transformation of $A_{m}$ (though it is, of course, present also in the equivalent set of equations that follow from (4.1)).

Combined with the type IIB effective action (3.13) the $D$-string action plays the role of a source $\left(X^{m}=x^{m}, X^{i}=0\right)$ for a set of fundamental string solutions of type IIB effective equations constructed in [33]. These conclusions are consistent with the previous results about $S L(2, Z)$ covariant family of type IIB strings [33,10] supporting the $S L(2, Z)$ duality symmetry of type IIB theory [8,9].

9 As was mentioned already, these actions are low-energy effective actions (analogous, e.g., to Nambu-type action for a cosmic string or domain wall) and may be treated semiclassically.

10 The parameter $\Lambda_{0}$ takes discrete values at the quantum level [10]. In what follows we shall often not differentiate between $S L(2, Z)$ and $S L(2, R)$ transformations. 


\subsection{D-2-brane}

In the case of $S_{3}$ (3.11) the Lagrange multiplier is $\Lambda^{m n}=\epsilon^{m n k} \partial_{k} \tilde{A}$ (cf.(2.3)) and thus elimination of $\mathcal{F}_{m n}$ (using, e.g., the representation (2.4) gives the dual action which is a generalisation of the expression in (2.6) (equivalent but less straightforward derivation of this action was given in [19])

$$
\begin{aligned}
\tilde{S}_{3}=\int d^{3} x[ & e^{-\phi} \sqrt{\operatorname{det}\left\{\hat{G}_{m n}+e^{2 \phi}\left(\partial_{m} \tilde{A}+\hat{C}_{m}\right)\left(\partial_{n} \tilde{A}+\hat{C}_{n}\right)\right\}} \\
& \left.+\frac{1}{2} i \epsilon^{m n k}\left(\frac{1}{3} \hat{C}_{m n k}-\hat{B}_{m n} \partial_{k} \tilde{A}\right)\right] .
\end{aligned}
$$

This action can be re-written in the standard membrane action form with one extra scalar coordinate $\tilde{A}$

$$
\begin{gathered}
\tilde{S}_{3}=\int d^{3} x\left(\sqrt{\operatorname{det} \hat{G}_{m n}^{\prime}}+\frac{1}{6} i \epsilon^{m n l} \hat{B}_{m n l}^{\prime}\right), \\
\hat{G}_{m n}^{\prime}=e^{-\frac{2}{3} \phi} \hat{G}_{m n}+e^{\frac{4}{3} \phi}\left(\partial_{m} \tilde{A}+\hat{C}_{m}\right)\left(\partial_{n} \tilde{A}+\hat{C}_{n}\right), \quad \hat{B}_{m n l}^{\prime}=\hat{C}_{m n l}-3 \hat{B}_{[m n} \partial_{l]} \tilde{A} .
\end{gathered}
$$

Dualising $A_{m}$ one thus finds an action which (for trivial background fields) has hidden global Lorentz symmetry $S O(1,10)$ of 11-dimensional theory as previously suggested in 49, 31. Because of the same underlying supersymmetry and field content (as implied by the dimensional reduction relation between $D=11$ supergravity and type IIA lowenergy effective action) it is not actually surprising to find that the $D$-2-brane action is dual to the direct dimensional reduction (fields do not depend on $X^{11} \equiv \tilde{A}$ ) of the $D=11$ supermembrane action coupled to $D=11$ supergravity background [18, 19]. The degrees of freedom count and the requirement of supersymmetry of a $D$-2-brane action in a background of $N=2 a, D=10$ supergravity uniquely fixes its form to be equivalent to that of the dimensionally reduced $D=11$ supermembrane action [34]. This observation can be used to determine [18 the fermionic terms in the $D$-membrane action (4.4) by starting with the known $D=11$ supermembrane action.

\section{3. $S L(2, R)$ self-duality of D-3-brane action}

Let us now turn to the case of our main interest - the D-3-brane action (3.12) and perform the world-sheet vector duality transformation $A_{m} \rightarrow \tilde{A}_{m}$ as in the case of the generalised $d=4 \mathrm{BI}$ action (2.11),(2.14). Adding the Lagrange multiplier term $\left(\Lambda^{m n}=\right.$ $\epsilon^{m n k p} \partial_{k} \tilde{A}_{p}$, cf. (2.3)) we find from (3.12)

$$
S_{4}=S_{4}^{\prime}+S_{4}^{\prime \prime}, \quad S_{4}^{\prime \prime}=\int d^{4} x \frac{1}{8} i \epsilon^{m n k l}\left(\frac{1}{3} \hat{C}_{m n k l}-2 \hat{B}_{m n} \tilde{F}_{k l}\right)+\ldots,
$$




$$
\begin{gathered}
S_{4}^{\prime}=\int d^{4} x\left[e^{-\phi} \sqrt{\operatorname{det}\left(\hat{G}_{m n}+\mathcal{F}_{m n}\right)}+\frac{1}{2} i \tilde{\Lambda}^{m n} \mathcal{F}_{m n}+\frac{1}{8} i \epsilon^{m n k l} C \mathcal{F}_{m n} \mathcal{F}_{k l}\right], \\
\tilde{\Lambda}^{m n} \equiv \epsilon^{m n k l}\left(\tilde{F}_{k l}+\hat{C}_{k l}\right), \quad \tilde{F}_{m n}=\partial_{m} \tilde{A}_{n}-\partial_{n} \tilde{A}_{m} .
\end{gathered}
$$

The remaining problem of eliminating $\mathcal{F}$ from $S_{4}^{\prime}$ is solved as in the case of BI action (2.11). Indeed, the action (3.12), (4.7) is closely related to (2.11) as can be seen by expressing it in terms of the $S L(2, R)$ invariant $D=10$ Einstein-frame metric $g_{\mu \nu}: \hat{G}_{m n}=e^{\frac{1}{2} \phi} \hat{g}_{m n}$. Then the dilaton dependence becomes the same as in (2.11) with $\hat{g}_{m n}$ replacing $\delta_{m n}$ there. As a result, the action dual to (3.12) is found to be 11

$$
\begin{gathered}
\tilde{S}_{4}=\int d^{4} x\left[e^{-\tilde{\phi}} \sqrt{\operatorname{det}\left(\hat{\tilde{G}}_{m n}+\tilde{\mathcal{F}}_{m n}\right)}\right. \\
\left.+\frac{1}{8} i \epsilon^{m n k l}\left(\frac{1}{3} \hat{C}_{m n k l}+2 \hat{\tilde{C}}_{m n} \tilde{\mathcal{F}}_{k l}+\tilde{C} \tilde{\mathcal{F}}_{m n} \tilde{\mathcal{F}}_{k l}\right)+\ldots\right], \quad \tilde{\mathcal{F}}_{m n}=\tilde{F}_{m n}+\hat{\tilde{B}}_{m n},
\end{gathered}
$$

where the transformed fields $\tilde{\phi}, \tilde{C}$ are as in (2.15) and

$$
\tilde{G}_{\mu \nu}=e^{\frac{1}{2}(\tilde{\phi}-\phi)} G_{\mu \nu}, \quad \tilde{B}_{\mu \nu}=C_{\mu \nu}, \quad \tilde{C}_{\mu \nu}=-B_{\mu \nu}
$$

As in the case of (2.14) these redefinitions of background fields correspond to the basic $S L(2, R)$ duality transformation (3.15) with $p=0, q=1, r=-1, s=0, \lambda \rightarrow-1 / \lambda$ and thus together with trivial shifts of $C$ imply the full $S L(2, R)$ invariance of the action under $A_{m} \rightarrow \tilde{A}_{m}$ combined with the transformation (3.15) of background fields.

The duality invariance of the $D$-3-brane action is thus closely related to the fact of self-duality of the $d=4 \mathrm{BI}$ action and its $S L(2, R)$ generalisation existing in the case of specific dilaton-axion-BI system first noted in 32.12

11 We assume that the action (3.12), (4.6) contains a specific 'higher - order' term $\sim i \epsilon^{m n k l}$ $\hat{B}_{m n} \hat{C}_{m n}$ as demanded by antisymmetric tensor gauge invariance.

12 It was found in [32] that there exists the unique generalisation of the $d=4 \mathrm{BI}$ action to the case of coupling to the dilaton $\phi$ and axion $C$ whose equations of motion are invariant under the $S L(2, R)$ duality transformations generalising the vector duality transformations: $S_{G R}=$ $\int d^{4} x\left[e^{-\phi} \sqrt{\operatorname{det}\left(e^{\frac{1}{2} \phi} g_{m n}+F_{m n}\right)}+\frac{1}{8} i C \epsilon^{m n k l} F_{m n} F_{k l}\right]$. This action (which is the same as (2.11) with $\left.\delta_{m n} \rightarrow g_{m n}\right)$ was combined in [32] with the standard $d=4$ axion-dilaton action $S_{\text {eff }}=$ $-\int d^{4} x \sqrt{g}\left[R-\frac{1}{2}(\partial \phi)^{2}-\frac{1}{2} e^{2 \phi}(\partial C)^{2}\right]$. The result $S=S_{e f f}+S_{G R}$ is not, however, the effective action that appears (upon dimensional reduction to $D=4$ ) in type I superstring theory (or that may appear [50] in $S O(32)$ heterotic string theory as suggested by its duality to type I theory in $D=10$ ): for $g_{m n}$ to be the Einstein-frame metric, its factor should be $e^{-2 \phi}$, not $e^{\frac{1}{2} \phi}$ as in $S_{G R}$. 
Thus we find that in contrast to the case of $S L(2, R)$ covariant $D$-string action, the $D$ 3-brane action is invariant under the $S L(2, R)$ transformations of the background fields of type IIB theory combined with world-volume vector duality. This conclusion is consistent with the expectation [33] that while there are $S L(2)$ multiplets of string (and 5-brane) solutions in type IIB theory, the self-dual 3-brane solution [1,:2] should be unique and related observation about the absence of bound states of $D$-3-branes was made in [10].

The supersymmetric self-dual 3-brane solution of type IIB effective field equations [2] (extreme limit of black 3-brane of [1]) has non-zero value of $C_{4}$ field $\left(C_{m n k l} \sim \epsilon_{m n k l} f\left(x^{i}\right)\right.$, $\left.\partial_{\left[i_{1}\right.} C_{\left.i_{2} i_{3} i_{4} i_{5}\right]} \sim \epsilon_{i_{1} i_{2} i_{3} i_{4} i_{5} i_{6}} \partial_{i_{6}} f\right)$ and curved metric. It should be possible to obtain this background as a solution of the combined action of type IIB low-energy field theory (3.13) and $D$-3-brane (3.12), i.e. it should be supported by the $D$-3-brane source. Assuming that all the background fields except the metric $G_{m n}$ and $C_{4}$ are trivial and that a consistent choice for the 3-brane fields is $X^{m}=x^{m}, X^{i}=0, A_{m}=0$, one finds that differentiating (3.12) over $C_{\mu \nu \lambda \kappa}$ produces a $\delta^{(6)}$-function source in the equation for $C_{4}, d^{*} d C_{4} \sim \delta^{(6)}\left(x^{i}\right)$. Since the action (3.13) is used under the prescription that that the resulting equations of motion should be consistent with self-duality of $F\left(C_{4}\right)$, one should also add the same source to the Bianchi identity $d d C_{4} \sim \delta^{(6)}\left(x^{i}\right)$. The self-duality equation for $d C_{4}$ then holds even in the presence of the 3-brane source. This prescription is in agreement with what follows from demanding the conformal invariance in the presence of 3-brane [15] and is also consistent with the interpretation of the solution [1,2] as having both 'electric' and

That remained a puzzle in [29,32]. As we have seen above, an action closely related to $S_{G R}$ does appear in string theory - as the action of $D$-3-brane of type IIB superstring theory. In ten (but not in four) dimensions $G_{m n}=e^{\frac{1}{2} \phi} g_{m n}$ is indeed the transformation from the string frame to the $S L(2, R)$ invariant Einstein-frame metric. The analogue of the invariant action $S=S_{\text {eff }}+S_{G R}$ is the sum of $D=10$ effective action $S_{\text {eff IIB }}(3.13)$ and 3-brane action (3.12) and the $S L(2, R)$ symmetry in question is not the usual $S$-duality of $d=4$ effective string action but $S L(2, R)$ duality of $D=10$ type IIB superstring theory. 
'magnetic' charges. 13 The resulting 3-brane field configuration is then the same as in [2]. 14

\section{Role of world-volume vector field and higher dimensional interpretations of $D$-branes of type II theories}

Let us draw some lessons from the above discussion of $D$-brane actions. One of the conclusions is that in the case of type IIB $D$-branes the duality transformation of the world-volume vector $A_{m}$ is closely related to (and, in fact, may be the origin of) the $S L(2, Z)$ symmetry of type IIB theory. The combined type IIB $+D$-brane effective action is covariant under $S L(2, Z)$ provided one also performs the duality rotation of $A_{m}$. This is strongly analogous to the relation between the world-sheet $d=2$ scalar duality and the target space duality symmetry of the string-theory effective action. In fact, the world-sheet duality symmetry is the reason why the string effective action is invariant under $T$-duality. The sum of string effective action and fundamental string source action is $T$-duality covariant provided the isometric string source coordinates are transformed simultaneously with the background fields. This analogy suggests that if the type IIB superstring theory could be interpreted also as a theory of fundamental supersymmetric 3branes, then its $S L(2, Z)$ symmetry would be a consequence of the $d=4$ 'electro-magnetic'

13 There should exist a more systematic approach to derivation of the full set of equations of motion from an action principle based on doubling of the R-R fields (cf. [39]). Then the original 'electric' $C_{4}$ and its 'magnetic' double $\bar{C}_{4}$ will appear in both the effective type II action and the $D$-brane action and should automatically lead to a consistent set of field equations with 'dyonic' sources. The dyonic nature of 3-brane is reflected also in the fact that the 3-brane source effectively drops out from the Einstein equations: for the 3-brane metric $d s^{2}=f^{-1 / 2} d x^{m} d x^{m}+f^{1 / 2} d x^{i} d x^{i}$ the $(m n)$ and $(i j)$ components of the Einstein equations $\left(R_{\mu \nu} \sim\left(F_{5}\right)_{\mu \nu}^{2}\right)$ reduce to $f^{-2} \partial^{i} \partial_{i} f=0$ and $f^{-1} \partial^{i} \partial_{i} f=0$ which are satisfied everywhere for $f=1+Q / r^{4}$ (equivalently, that means that the 3 -brane source $\delta$-function will be multiplied by a power of $r$ near $r=0$ ). In contrast to the case of other 'elementary' $p$-brane solutions here the same conclusion applies also to the Einstein equations with raised indices (as they follow from the effective action). This may be related to non-singularity of the black 3-brane solution [51.

14 It was found in [2] that the supersymmetric 3-brane solution preserves half of $N=2 b, D=10$ supersymmetry and thus should be described by $8+8$ on-shell degrees of freedom of $d=4, N=4$ Maxwell supermultiplet $\left(A_{m}, X^{I J}, \lambda^{I}\right)$. Since 4 other supersymmetries are spontaneously broken, they should be realised in a non-linear way, so it was conjectured that the resulting action should be of an unusual Born-Infeld type [2]. This conjecture is indeed confirmed by the above $D$-brane construction of the 3-brane action. 
world-volume vector duality. Indeed, just as the string partition function in an isometric background is $T$-duality invariant (since the string coordinates are integrated out, their transformation is irrelevant), the fundamental 3-brane partition function in type IIB theory background will be invariant under the $S L(2, Z)$ transformations (3.14) (since the vector field $A_{m}$ is an integration variable, the vector duality transformation $A_{m} \rightarrow \tilde{A}_{m}$ which accompanies (3.14) is irrelevant).

The vector duality plays a different role in the case of type IIA $D$-2-brane: it reveals a hidden $O(1,10)$ Lorentz symmetry making possible to interpret the resulting action is a dimensional reduction of the $D=11$ supermembrane action [18,19]. This implies a special role played by the $D$-2-brane in type IIA theory. The above remarks suggest that $D$-3-brane plays analogous special role in type IIB theory.

Similarity with 2-brane case suggests that the dimension 'associated' with $D$-3-branes is 12 . Indeed, recall that the $D$-p-branes of type II theories are described by the the reparametrisation invariant actions depending on the fields $X^{\mu}, A_{m}(\mu=0, \ldots, 10 ; m=$ $0, \ldots, d-1 ; d=p+1)$. As was mentioned already, the number of the physical degrees of freedom is the same for all values of $d=p+1: 10-d+d-2=8$, where $10-d$ is the number of transverse coordinates $X^{i}$ remaining, e.g., in the static gauge $X^{m}(x)=x^{m}$ and $d-2$ is the number of transverse modes of a vector in $d$ with the standard $U(1)$ Maxwell kinetic term (leading order term in the expansion of (3.9)).15 Suppose we fix the $U(1)$ gauge but keep the reparametrisation invariance with an idea to reinterpret the $D$-p-brane action as another reparametrisation invariant $p$-brane action. Then the number of 'partially off-shell' bosonic degrees of freedom becomes $10+d-2=9+p$. This gives ten for a string, 16 eleven for the 2-brane, twelve for 3-brane, etc. At the same time,

15 Equivalently, one may fix reparametrisation invariance by choosing $A_{m}=f_{m}(x)$ to be some given functions but then one gets -2 as $U(1)$ ghost contributions.

16 The critical dimension of the $D$-string should not, of course, change from its standard (10 or 26) value, in spite of the presence of an extra $U(1)$ vector field in its action. Indeed, let us consider for simplicity the purely bosonic case. The fundamental $D$-string is described by a formal path integral $Z=\int[d X][d A] \exp \left[-T \int d^{2} x \sqrt{\operatorname{det}\left(g_{m n}+F_{m n}\right)}\right]$, where $g_{m n}=\partial_{m} X^{\mu} \partial_{n} X^{\mu}$ is also used in the definition of the measure $[d x][d A]$. As in the Nambu string case, to make this path integral tractable one needs to add some auxiliary fields (like independent $2 \mathrm{~d}$ metric in Polyakov approach). Since we are interested here in the effect of integrating out $A_{m}$, it is sufficient to introduce just one auxiliary field $V$ as in (2.4), defining $Z$ as (cf.(2.4),(2.5)) $Z=$ $\int[d X][d V][d A] \exp \left[-\frac{1}{2} T \int d^{2} x \sqrt{\operatorname{det} g_{m n}}\left[V\left(1+\frac{1}{2} F_{m n} F^{m n}\right)+V^{-1}\right]\right.$. Integrating out $A_{m}$ we do not get any non-trivial determinants (the $U(1)$ ghost contribution cancels out for the same reason why a propagating $2 \mathrm{~d}$ vector field has zero degrees of freedom). We are then left with an action which is (semiclassically) equivalent to the Nambu action and thus has the same critical dimension 26. 
the amount of on-shell supersymmetry should still remain the same for all $p$-branes and their 'higher-dimensional' versions (there are 8 fermionic physical degrees of freedom as demanded by unbroken supersymmetry [7]).

This indeed is what happens in the case of the 2 -brane $-D=11$ membrane connection [18]. Since the vector is dual to a scalar in $d=3$ but to a vector in $d=4$, in the case of 3-brane the two extra degrees are not easily combined with $10 X^{\mu}$ scalar fields in a $D=12$ Lorentz-invariant way. Thus if type IIB superstring theory admits a 3-brane reformulation, its 12-dimensional interpretation should be more subtle than the 11-dimensional one of type IIA theory implied by the $D$-2-brane - supermembrane connection: there should be no simple $D=12$ Lorentz group relating all of the corresponding $(10+2)$ bosonic degrees of freedom. This suggests that the two extra dimensions (effectively represented by the 'transverse' part of $A_{m}$ ) may play only an auxiliary role in some novel realisation of $D=10$ supersymmetry.

Suggestions about possible relation of type IIB theory to a 12-dimensional theory were previously made also in [35, 36, 37]. It was conjectured in [35] that there may exist a 3-brane with world-sheet signature $(2,2) \frac{17}{17}$ moving in a $D=12$ space with signature $(2,10)$ which reduces upon double-dimensional reduction to the usual type IIB superstring just like the $D=11$ supermembrane reduces to the type IIA string [52] (this was motivated by the fact that in the case of $(2,10)$ signature there exist both Majorana-Weyl spinors and self-dual tensors in the supersymmetry algebra). A picture of $(2,2)$ world-volumes embedded in $(2,10)$ space independently emerged in the context of $N=2$ string theory [53] (where the 'transverse' 8-dimensional space was treated as an internal one, compactified on a special torus). The existence of $D$-string in type IIB theory with an extra vector field on the world sheet was used in [37 to conjecture (by analogy with $N=2$ strings where there is a local $U(1)$ gauge symmetry on the world-sheet) that there may exist an 'off-shell' extension of type IIB theory where two extra compact $(1,1)$ dimensions are added both to the worldsheet and the target space. Type IIB strings would then be recovered by wrapping the $(1,1)$ part of world-volume around the compact $(1,1)$ part of the target space as in [35]. A possible 12-dimensional origin of $S L(2, Z)$ symmetry of type IIB theory was conjectured in [36] (were the signature of a $D=12$ space-time was assumed to be the standard one). These conjectures may be related to the systematic approach [54 to a unified description of different world-sheet string (and membrane) theories as effective target space theories [55]

17 The 4-parameter diffeomorphism invariance is, in principle, sufficient to gauge-fix more than one time-like direction. Ref. [35] discussed various $p$-branes with several time-like coordinates. 
of $N=(2,1)$ heterotic string. Here the central role is played by $(2,2)$ self-dual geometries embedded in $(2,10)$ space (with non-compact transverse string coordinates appearing as zero modes of $N=(2,1)$ string).

Suggestions of a formulation based on $(2,2)$ world-volumes in $(2,10)$ space-time are apparently different from what was found above for the $D$-3-brane. Since all 8 transverse degrees of freedom $X^{i}$ and $A_{m}^{\perp}$ have the same signs of kinetic terms it seems natural to assign the standard spatial signature to the extra two dimensions. Also, the $D$-3-brane has standard $(1,3)$ world-volume signature (in fact, all $D$-branes have $(1, p)$ signature). Finally, one should not expect to find the full 12-dimensional Lorentz (super)symmetry. It would be interesting to see if the $(1,11)+(1,3)$ picture directly implied by the $D$-3-brane description can still be related to the $(2,10)+(2,2)$ proposals. 18

To conclude, it is likely that the self-dual Dirichlet 3-brane plays a special role in type IIB superstring theory, explaining its $S L(2, Z)$ symmetry in terms of the world-volume 'electro-magnetic' duality and also pointing towards an unusual non Lorentz invariant 12-dimensional reformulation of this theory. Absence of manifest Lorentz symmetry is characteristic to models which describe chiral $d$-forms [56,57] and also to models with doubled numbers of field variables (but the same number of degrees of freedom) which are manifestly duality invariant [38,39]. It may be that 12-dimensional theory is related to such 'doubled' formulation of a self-dual theory.

\section{Acknowledgements}

I would like to thank G. Gibbons, C. Schmidhuber and P.K. Townsend for stimulating discussions. I acknowledge also the support of PPARC, ECC grant SC1*-CT92-0789 and NATO grant CRG 940870.

18 To relate the $(1,3)$ and $(2,2)$ theories one presumably needs to perform a transformation analogous to interchanging the left and right string modes for a time-like circle as in [37] (I am grateful to C. Vafa for this suggestion). In addition, to relate $(1,11)$ and $(2,10)$ descriptions one needs to 'twist' the vector field degrees of freedom. Twisting of $4 \mathrm{~d}$ vector field may be analogous to twisting of $2 \mathrm{~d}$ chiral scalars: in the 'doubling' approach to construction of manifestly dual actions [38, 39] the $d=2$ scalar and $d=4$ vector Lagrangians have similar structure $L_{d}(\mathcal{A})=$ $-\frac{1}{2} \partial_{0} \mathcal{A L} \partial \mathcal{A}+\frac{1}{2} \partial \mathcal{A} \mathcal{M} \partial \mathcal{A}$, where $\mathcal{A}=(A, \tilde{A}), \quad A$ and $\tilde{A}$ are $k$-forms (spatial parts of gauge potentials), $k=\frac{1}{2}(d-2)$, and $\mathcal{L}=\left(\begin{array}{cc}0 & I \\ (-I)^{k} & 0\end{array}\right), \mathcal{M}=\left(\begin{array}{ll}I & 0 \\ 0 & I\end{array}\right)$. 


\section{References}

[1] G. Horowitz and A. Strominger, Nucl. Phys. B360 (1991) 197.

[2] M.J. Duff and X. Lu, Phys. Lett. B273 (1991) 409.

[3] M.J. Duff, R. Khuri and X. Lu, Phys. Repts. 259 (1995) 213.

[4] J. Dai, R.G. Leigh and J. Polchinski, Mod. Phys. Lett. A4 (1989) 2073.

[5] R.G. Leigh, Mod. Phys. Lett. A4 (1989) 2767.

[6] M.B. Green, Phys. Lett. B329 (1994) 435.

[7] J. Polchinski, Phys. Rev. Lett. 75 (1995) 4724, hep-th/9510017.

[8] C.M. Hull and P.K. Townsend, Nucl. Phys. B438 (1995) 109.

[9] E. Witten, Nucl. Phys. B443 (1995) 85.

[10] E. Witten, IASSNS-HEP-95-83, hep-th/9510135.

[11] M. Li, BROWN-HET-1020, hep-th/9510161.

[12] I.R. Klebanov and L. Thorlacius, PUPT-1574, hep-th/9510200; S.S. Gubser, A. Hashimoto, I.R. Klebanov and J.M. Maldacena, PUPT-1586, hep-th/9601057.

[13] C. Bachas, NSF-ITP/95-144, hep-th/9511043.

[14] C.G. Callan and I.R. Klebanov, PUPT-1578, hep-th/9511173.

[15] M. Douglas, RU-95-92, hep-th/9512077.

[16] J. Polchinski, S. Chaudhuri and C.V. Johnson, NSF-ITP-96-003, hep-th/9602052.

[17] M.B. Green, DAMTP/96, hep-th/9602061.

[18] P.K. Townsend, DAMTP-R/95/59, hep-th/9512062.

[19] C. Schmidhuber, PUPT-1585, hep-th/9601003.

[20] S.P. de Alwis and K. Sato, COLO-HEP-368, hep-th/9601167.

[21] E.S. Fradkin and A.A. Tseytlin, Phys. Lett. B163 (1985) 123.

[22] A. Abouelsaood, C. Callan, C. Nappi and S. Yost, Nucl. Phys. B280 (1987) 599.

[23] E. Bergshoeff, E. Sezgin, C.N. Pope and P.K. Townsend, Phys. Lett. B188 (1987) 70.

[24] R.R. Metsaev, M.A. Rahmanov and A.A. Tseytlin, Phys. Lett. B193 (1987) 207; A.A. Tseytlin, Phys. Lett. B202 (1988) 81.

[25] O.D. Andreev and A.A. Tseytlin, Phys. Lett. B207 (1988) 157; Nucl. Phys. B311 (1988) 205.

[26] C.G. Callan, C. Lovelace, C.R. Nappi and S.A. Yost, Nucl. Phys. B308 (1988) 221.

[27] E. Schrödinger, Proc. Roy. Soc. A150 (1935) 465.

[28] H.C. Tze, Nuov.Cim. 22A (1974) 507.

[29] G.W. Gibbons and D.A. Rasheed, Nucl. Phys. B454 (1995) 185, hep-th/9506035.

[30] J.H. Schwarz, Nucl. Phys. B226 (1983) 269; P.S. Howe and P.C. West, Nucl. Phys. B238 (1984) 181.

[31] M.J. Duff, J.T. Liu and R. Minasian, Nucl. Phys. B452 (1995) 261; hep-th/9506126.

[32] G.W. Gibbons and D.A. Rasheed, Phys. Lett. B365 (1996) 46, hep-th/9509141. 
[33] J.H. Schwarz, Phys. Lett. B360 (1995) 13; B364 (1995) 252 (E), hep-th/9508143, hep-th/9509148; CALT-68-2025, hep-th/9510086.

[34] E. Bergshoeff, E. Sezgin and P.K. Townsend, Phys. Lett. B189 (1987) 75; Ann. of Phys. 185 (1988) 330.

[35] M. Blencowe and M.J. Duff, Nucl. Phys. B310 (1988) 387.

[36] C.M. Hull, QMW-95-50, hep-th/9512181.

[37] C. Vafa, HUTP-96/A004, hep-th/9602022.

[38] A.A. Tseytlin, Phys. Lett. B242 (1990) 163; Nucl. Phys. B350 (1991) 395.

[39] J.H. Schwarz and A. Sen, Nucl. Phys. B411 (1994) 35; Phys. Lett. B312 (1993) 105.

[40] M. Born and L. Infeld, Proc. Roy. Soc. A144 (1934) 425.

[41] E. Bergshoeff, L.A.J. London and P.K. Townsend, Class. Quantum Grav. 9 (1992) 2545.

[42] A.A. Tseytlin, Phys. Lett. B251 (1990) 530.

[43] E.S. Fradkin and A.A. Tseytlin, Phys. Lett. B158 (1985) 316; Phys. Lett. B160 (1985) 69.

[44] A.A. Tseytlin, Phys. Lett. B208 (1988) 221; Int. J. Mod. Phys. A4 (1989) 1257.

[45] R.R. Metsaev and A.A. Tseytlin, Nucl. Phys. B298 (1988) 109; A.A. Tseytlin, Int. J. Mod. Phys. A5 (1990) 589.

[46] C. Callan, D. Friedan, E. Martinec and M. Perry, Nucl. Phys. B262 (1985) 593.

[47] E. Bergshoeff, C.M. Hull and T. Ortín, Nucl. Phys. B451 (1995) 547, hep-th/9504081.

[48] E. Bergshoeff, H.J. Boonstra and T. Ortín, UG-7/95, hep-th/9508091.

[49] M.J. Duff and J.X. Lu, Nucl. Phys. B390 (1993) 276, hep-th/9207060.

[50] A.A. Tseytlin, Phys. Lett. B367 (1996) 84, hep-th/9510173

[51] G.W. Gibbons, G.T. Horowitz and P.K. Townsend, Class. Quant. Grav.12 (1995) 297, hep-th/9410073.

[52] M.J. Duff, P.S. Howe, T. Inami and K.S. Stelle, Phys. Lett. B191 (1987) 70.

[53] H. Ooguri and C. Vafa, Nucl. Phys. B361 (1991) 469; Nucl. Phys. B367 (1991) 83.

[54] D. Kutasov and E. Martinec, EFI-96-04, hep-th/9602049.

[55] M.B. Green, Nucl. Phys. B293 (1987) 593.

[56] N. Marcus and J.H. Schwarz, Phys. Lett. B115 (1982) 111.

[57] R. Floreanini and R. Jackiw, Phys. Rev. Lett. 59 (1987) 1873; M. Henneaux and C. Teitelboim, Phys. Lett. B206 (1988) 650. 